\title{
Coleridge: a computer tool for assisting musical reflection and self-explanation
}

\author{
John Cook* and Nigel Morgan** \\ *University Centre for Complementary Learning, Thames Valley University \\ **Department of Music, Bretton Hall University College
}

This paper examines some of the problems involved when learning how to compose music A prototype computer-based music tool called Coleridge is described. Coleridge was used in a study that investigated the dialogues that took place when a mentor attempted to encourage creative reflection in students. Results of dialogue analysis suggested that because learners seem unable to make accurate predictions about how a musical phrase will sound, there is a real need for a computer-based learning assistant. Finally, the paper reports on how these findings were used to motivate the design of a mentor's assistant in a new version of Coleridge.

\section{Introduction}

Since the mid-1980s, there has been a movement away from knowledge supplied by the teacher and towards talking, reflecting and explaining as ways to learn. An example of this change in focus is provided by the self-explanation work of Chi et al (1994) who describe an approach to talking science rather than hearing science. According to Chi and coworkers, generating explanations to oneself (self-explanations) facilitates the integration of new information into existing knowledge. Reflecting about one's own learning is the same as thinking about learning or metacognition. Metacognition can be defined as the understanding of knowledge, an understanding that can be reflected in either effective use or overt description of the knowledge in question (Brown, 1987). This definition of metacognition requires of a learner both internalized thinking about learning (that is, reflection), and externalized communication, through language or action, that indicates an understanding of knowledge (that is, a self-explanation). In the work described in this paper the overall pedagogical goal is to encourage creative reflection in learners. Creative reflection is defined as the ability of a learner to imagine musical opportunities in novel situations, and then to make accurate predictions (verbally) about these opportunities. To succeed at creative reflection there should be a correspondence between what a learner predicts will happen and what actually happens. An example would be a learner first writing a musical phrase using musical notation, then predicting verbally how that phrase will sound, playing the phrase back on a piano, and finally evaluating if the prediction was 
accurate or not. Very little work has been done on how computers can be used to support talking, reflecting and explaining in the creative subject-area of musical composition. The rest of this paper addresses this issue.

\section{The problem of learning how to compose music}

This section examines two aspects of the problem of thinking about how to compose music.

1. There is thinking that reflects upon creative intention and execution (creative thinking).

2. There is thinking in the monitoring of intention and execution (critical thinking) that reflects upon philosophical areas (meaning, experience, possibilities, aesthetic relationships).

Creative thinking in music composition has been challenged by the widespread use of sequencers (software recorders of performance data). These enable musical material to be assembled layer by layer. A playing, listening, editing process ensues, relieving the composer of the need to memorize and internalize successively imagined vertical positioning of musical material. Traditionally, however, the craft of music composition has always required the development of memory, reflection, critical review and analysis, all skills that focus on the higher-order patterning of musical material.

It is the perception of the authors of this paper that the current training of composers in higher education seems now beleaguered by a very contemporary problem: how to encourage and promote creative reflection in a culture where technology appears to provide a side-step for the disciplines of higher-order thinking. We also see musical formalism (Landy, 1997) itself being challenged by a more direct relationship with sound making, design and control. This puts the creation of performance directly in the hands of the composer; eschewing any need for the written musical score from which formal abstractions and the glue of syntax can be identified and reflected upon.

Critical thinking in the act of music composition is usually embedded in the further reflection (monitoring) after execution. It must deal with judgement, enable selfcorrection, and be sensitive to context. The strong element of intuitive action present in the process of composition (Sloboda, 1986) is invariably followed or accompanied by a monitoring activity. This focuses on either the sound itself being replayed in real time or on a formalism that becomes apparent from a kind of fast visual scanning in which the composer imagines rather than physically hears the music happen.

If we examine one aspect of critical thinking, we find that in music, meaning (for example) has to be built up successively. Centuries of composers' hand-written sketches show how they accrue representations of musical meaning using strategies that act on musical ideas in a cumulative and evolving fashion, and play with ambiguity, while keeping resolutions on decision-making on hold for long as possible. This is elegantly demonstrated in the sketchbooks of Beethoven and Stravinsky.

For the poet (and the reader), however, meaning is established almost instantaneously, as the words hit the page. And, as thinking in a creative writing class is perpetually bound to reading, speaking and writing (Lipman, 1991), the student writer becomes practised in 
engaging in both descriptive dialogues, reflective thinking and explanation. The meaning of words is normally associated with usage; rarely can a word have meaning on its own. The same is true for any musical parameter, for example the single note. But, an aggregate of notes, like a syntactical arrangement of words, can be said to have meaning by virtue of causal and contextual relationships. Regrettably, musicians are rarely trained to talk, reflect upon or explain such views of meaning without resorting to the formidable tools of musical analysis that are usually devoid of philosophical content.

\section{Prototype of Coleridge}

The system developed for and used in the study described below was a prototype called Coleridge (see Figure 1 for an annotated screen shot).

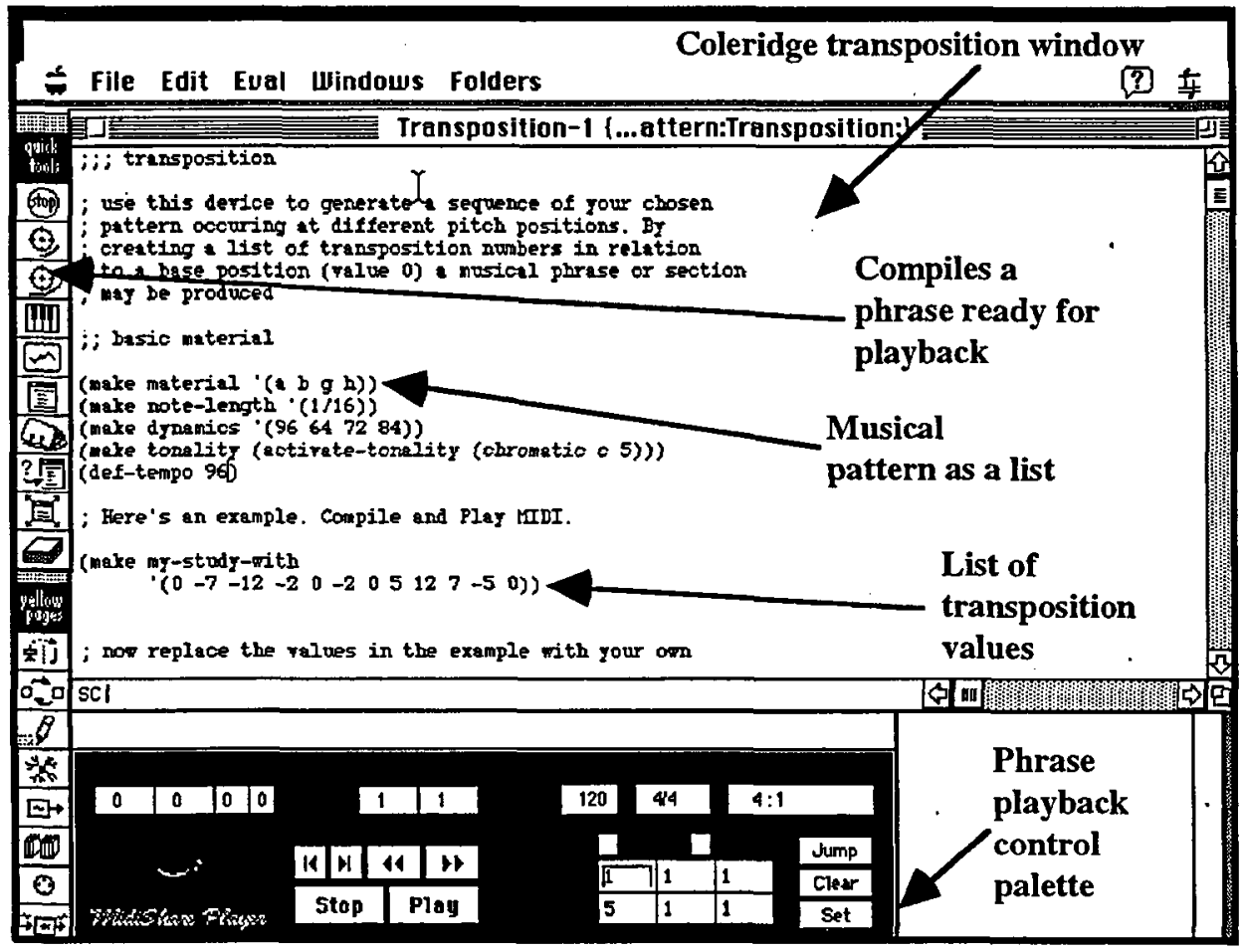

Figure 1: Annototed screen-shot of prototype Coleridge system used in the study.

The prototype Coleridge was designed to provide a single-step, fast-action playback of musical ideas. This would, we predicted, enable descriptive talk (that is, verbal representations of meaning) as well as reflective thought (self-monitoring) and explanation (accurate predictions plus more self-monitoring) to take place.

Coleridge was implemented in a Common Lisp-based music composition language called Symbolic Composer (Morgan and Tolonen, 1995). The prototype version of Coleridge was a constrained environment in that it dealt with only one small aspect of musical 
composition (the transformation of a musical pattern into a phrase or section). The technique used in Coleridge for transposing an initial pattern (for example, C C\#F\#) is simply to use a transposition number (which represent semi-tone steps, i.e. pitch transposition). By creating a list of transposition numbers in relation to a base position (value 0 ), a musical phrase or section may be produced. Zero will just simply give a repetition of the pattern (C C $\# \# G$ ), -7 gives a transposed-down repetition of the pattern (in this example F F\# B C).

\section{Study of musical reflecting and explaining}

The study took place in November 1996 using the prototype Coleridge described above. The study involved individual students discussing with a teacher-mentor (one of the authors of this paper) their attempts at creative reflection. The aim of the study was to answer the following research question: what are the interactive means by which a musiccomposition teacher stimulates creative reflection? For full details of the study and the dialogue analysis approach, which was based on goal hierarchies and updated speech-act theory, readers are encouraged to consult Cook (1997). Briefly, interactions were captured on video and transcriptions of the sessions were then analysed for occurrences of subgoals that relate to creative reflection. Scores for each subgoal were then generated.

Results from the analysis showed that students did not seem able to make accurate predictions, in spite of mentor support and a computer tool design to assist this process. Students did make some attempts at making a prediction, but only one out of four learners met with success in terms of accuracy. Other results showed that some internalized reflection in the form of self-monitoring took place.

The dialogue analysis approach recognized two self-monitoring subgoals. Monitoringevaluate was dialogue that involved some evaluative comment by the learner about the match between a prediction and an outcome. Monitoring-diagnose was an attempt by the learner to diagnose why something did or did not work. This finding is encouraging in that mentoring and Coleridge seemed to promote the monitoring effect, which we would claim is the first step towards creative reflection, and in particular the ability to make accurate predictions. If the students in the study had been allowed to take a second session, we would estimate that the score for making an accurate prediction would increase. The ability to make an accurate prediction is clearly difficult and may require repeated practice and guidance. However, in a post-experimental interview the mentor did point out that 'meeting this learning need may be a task that a human may have trouble providing justification for in the UK's busy curriculum'. It is therefore claimed that there is a real learning need for a computer-based mentor's assistant for Coleridge.

\section{A solution: the mentor's assistant}

This section describes the changes made to Coleridge (the new version is shown in Figure 2 ) in an attempt to act on the findings of the study described above. From a learner's perspective, Coleridge has been designed to assist independent practice in creative reflection. For the mentor, the computer tool is intended to be used as an assistant that promotes in learners the self-monitoring effect.

To enhance practice at creative reflection, Coleridge now offers student and mentor 


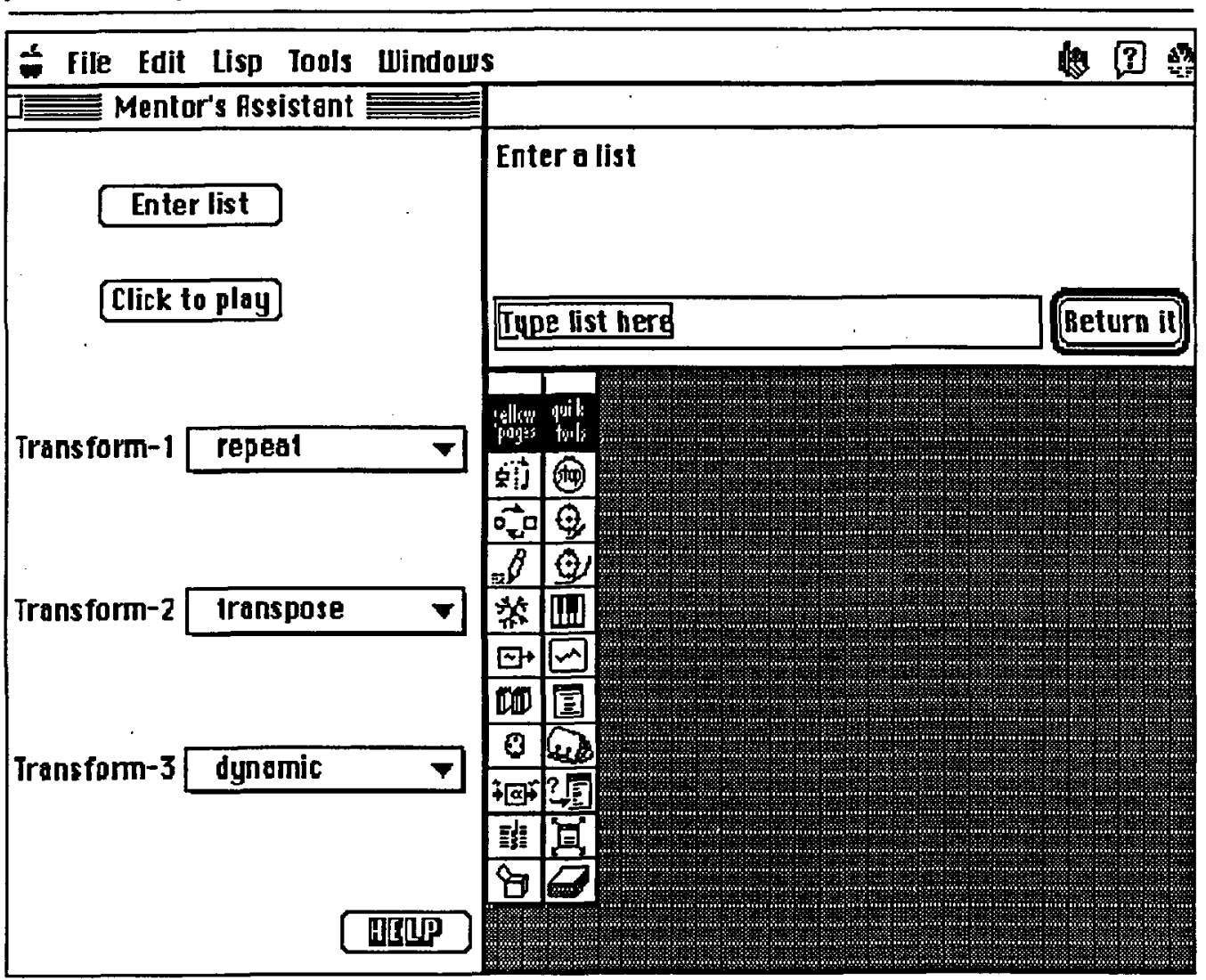

Figure 2: Coleridge with new interface and increased functionality.

opportunities to develop cumulative transformations (that is, several alterations) and parametric additions (for example, add some dynamics) to an initial phrase. Thus, if the learner clicks on the Enter list button shown in Figure 2, a dialogue box appears asking the learner to enter a list and to Return it (top-right of Figure 2). Only this, the first dialogue box, remains on the screen (learners are being encouraged to memorize the changes they make to a phrase). A good starting point would be to enter five zeros, return it, then to use the Click to play button to hear the start-up motive (C C F F $F$ ) repeated five times. This flat phrase can be developed by selecting the Transform-1 pop-up menu.

The learner is then presented with a list of what we call modifying processors. Modifying processors are the parameters available to a composer. The learner can select from eight modifying processors: dynamics, repeat, transposition, making pauses, hocketing, making variants (shuffle, reverse, rotate), octave displacements, and changing rhythm. In Figure 2, the learner has selected 'repeat', and will have been asked by a dialogue box to enter a repeat list. Repeat works as follows: 4 repeats the whole motive, 3 repeats only the first three notes leaving a pause for the fourth note, and so on down to 0 which leaves a pause where the four notes should have been played. Coleridge will then prompt the learner to predict verbally (to themselves or to another student) how the phrase will sound when it is 
played back. By using the Click to play button, the learner can then hear what the musical phrase sounds like with the first transformation applied.

A few seconds after playback, Coleridge will prompt the learner verbally to self-monitor their attempts at creative reflection (a message - Was that what you expected? - is displayed). If the Transform-2 pop-up menu is then selected, the learner will be offered the same list of eight modifying processors. The big difference here is that once a selection is made and data is entered via a dialogue box, the modification processor acts on the output from Transform-1 (i.e. it is a parametric addition). For example, in Figure 2 the learner has selected to transpose the output from modifying process Transform-1 (that is, repeat). The output from Transform- 2 can then be used as an input to Transform-3 to build up a phrase that makes use of three different modifying processes. Each time learners input a data list for a modifying process, they are encouraged to make a verbal prediction, and, following playback, verbally to self-monitor.

These three modification processes (Transform 1, 2 and 3) produce an engaging musical result, and one much closer to the composing of a musical phrase as might be part of the experience of a composer. Furthermore, we claim that with the addition of these modifiers and prompts for verbal predictions and self-monitoring, Coleridge has become a powerful and useful tool for student and mentor alike to progress talking, reflecting and explaining.

\section{Future work}

Future work will examine how to introduce some level of interaction with a collection of philosophical strands (Lipman, 1991), for example relating (music) to experience, art and craft, and aesthetical relationships. This should further promote the development of creative and critical thinking, and hence support directly the challenging aspects of mentoring.

\section{Acknowledgements}

Thanks to Michael Baker and Theresa Kinnison for their help with this paper.

\section{References}

Brown, A. (1987), 'Metacognition, executive control, self-regulation, and other mysterious mechanisms' in Weinert, F. and Kluwe, R. (eds), Metacognition, Motivation, and Understanding, Hillsdale, NJ: Lawrence Erlbaum, 65-116.

Chi, M., de Leeuw, N., Chiu, M.-H. and LaVancher, C. (1994), 'Eliciting self-explanations improves understanding', Cognitive Science, 18, 439-77.

Cook, J. (1997), 'Mentoring, metacognition and music: an analysis of the interactions used to support creative reflection' in Du Boulay, B. and Mizoguchi, R. (eds), Artificial Intelligence in Education: Knowledge and Media in Learning Systems. Proceedings of AI-ED 97 World Conference on Artificial Intelligence in Education, Kobe, Japan, Amsterdam: IOS Press, 271-8.

Landy, L. (1997), 'Editorial', Organised Sound, 2 (1), 1-3.

Lipman, M. (1991), Thinking in Education, Cambridge: CUP. 
John Cook and Nigel Morgon Coleridge: a computer tool for assisting musical reflection and self-explanation

Morgan, N. and Tolonen, P. (1995), Symbolic Composer Professional (software application for Apple Macintosh computers), Amsterdam: Tonality Systems.

Sloboda, J. (1985), The Musical Mind: The Cognitive Psychology of Music, Oxford: OUP. 